[RADIOCARBON, VOL 28, No. 2B, 1986, P 939-941]

\title{
HIGH-PRECISION CALIBRATION OF THE RADIOCARBON TIME SCALE, 3930-3230 CAL BC
}

AFM de JONG*, BERND BECKER**, and WG MOOK

Isotope Physics Laboratory, University of Groningen, The Netherlands

This paper contains ${ }^{14} \mathrm{C}$ results obtained by the special high-precision proportional gas counter, designed by Tans and Mook (1978), on tree rings from the South German oak chronologies: Donau 7, 3, 10, and 12, originating from the Danube River basin $\left(48^{\circ} 24^{\prime} \mathrm{N}, 10^{\circ} 5^{\prime} \mathrm{E}\right)$.

${ }^{4} \mathrm{C}$ and ${ }^{13} \mathrm{C}$ results were published earlier (De Jong, Mook \& Becker, 1979; De Jong \& Mook, 1980; De Jong, 1981). However, the historical time scale was then given as a floating series of tree-ring numbers, while an estimate of absolute scale was derived from wiggle-matching with the bristlecone curve of Suess (1978). Since the absolute Hohenheim master chronology could be extended to include this period (Linick, Suess \& Becker, 1985) we can attach absolute values to the historical scale. Because the resulting curve of Figure 1 is to be used as a ${ }^{14} \mathrm{C}$ calibration curve, we presented this historical time scale in cal BC.

The tree-ring samples (ca $100 \mathrm{~g}$ ) were pretreated according to the AAA procedure, consisting of 1 ) extraction with $4 \%$ hydrochloric acid solution at $80^{\circ} \mathrm{C}$ for 24 hours to remove resinous material; 2) extraction with $4 \%$ sodium hydroxide solution at $80^{\circ} \mathrm{C}$ for 24 hours to remove tannic acids; 3 )

* Present address: University of Utrecht, The Netherlands
** Universität Hohenheim, West Germany treatment with $4 \%$ hydrochloric acid solution at $80^{\circ} \mathrm{C}$ for several hours to remove atmospheric carbon dioxide, possibly absorbed during step 2 .

After each step, the samples were thoroughly washed with demineralized water to $\mathrm{pH}=7$. Further technical details were given by De Jong (1981).

The ${ }^{14} \mathrm{C}$ results, corrected for ${ }^{13} \mathrm{C}$, are presented in Table 1 and Figure 1 , where the thin lines refer to $1 \sigma$ values from counting statistics only, the other contributions being negligibly small.

\section{REFERENCES}

Becker, B and Kromer, B, 1986, Extension of the Holocene dendro-chronology by Preborea pine series, 8800 to $10,000 \mathrm{BP}$, in Stuiver, $\mathrm{M}$ and Kra, RS, eds, Internatl ${ }^{14} \mathrm{C}$ conf, 12 th Proc: Radiocarbon, this issue.

De Jong, A F M, (ms) 1981, Natural ${ }^{14} \mathrm{C}$ variations: PhD thesis, Univ Groningen.

De Jong, A F M and Mook, W G, 1980, Medium-term atmospheric ${ }^{14} \mathrm{C}$ variations, in Stuiver, $\mathrm{M}$ and Kra, RS, eds, Internatl ${ }^{14} \mathrm{C}$ Conf, 10th, Proc: Radiocarbon, 22, no. 2, p 267-272.

De Jong, A F M, Mook, W G and Becker, B, 1979, Confirmation of the Suess wiggles: 3200 3700 BC: Nature, v $280, p$ 48-49.

Linick, T W, Suess, H E and Becker, B, 1985, La Jolla measurements of radiocarbon in south German oak tree-ring chronologies: Radiocarbon, v 27, p 20-32.

Suess, H E, 1978, La Jolla measurements of radiocarbon in tree-ring dated wood: Radiocarbon, v 20, p $1-18$

Tans, P P and Mook, W G, 1978, Design, construction, and calibration of a high accuracy carbon-14 counting set up: Radiocarbon, v 21, p 22-40. 


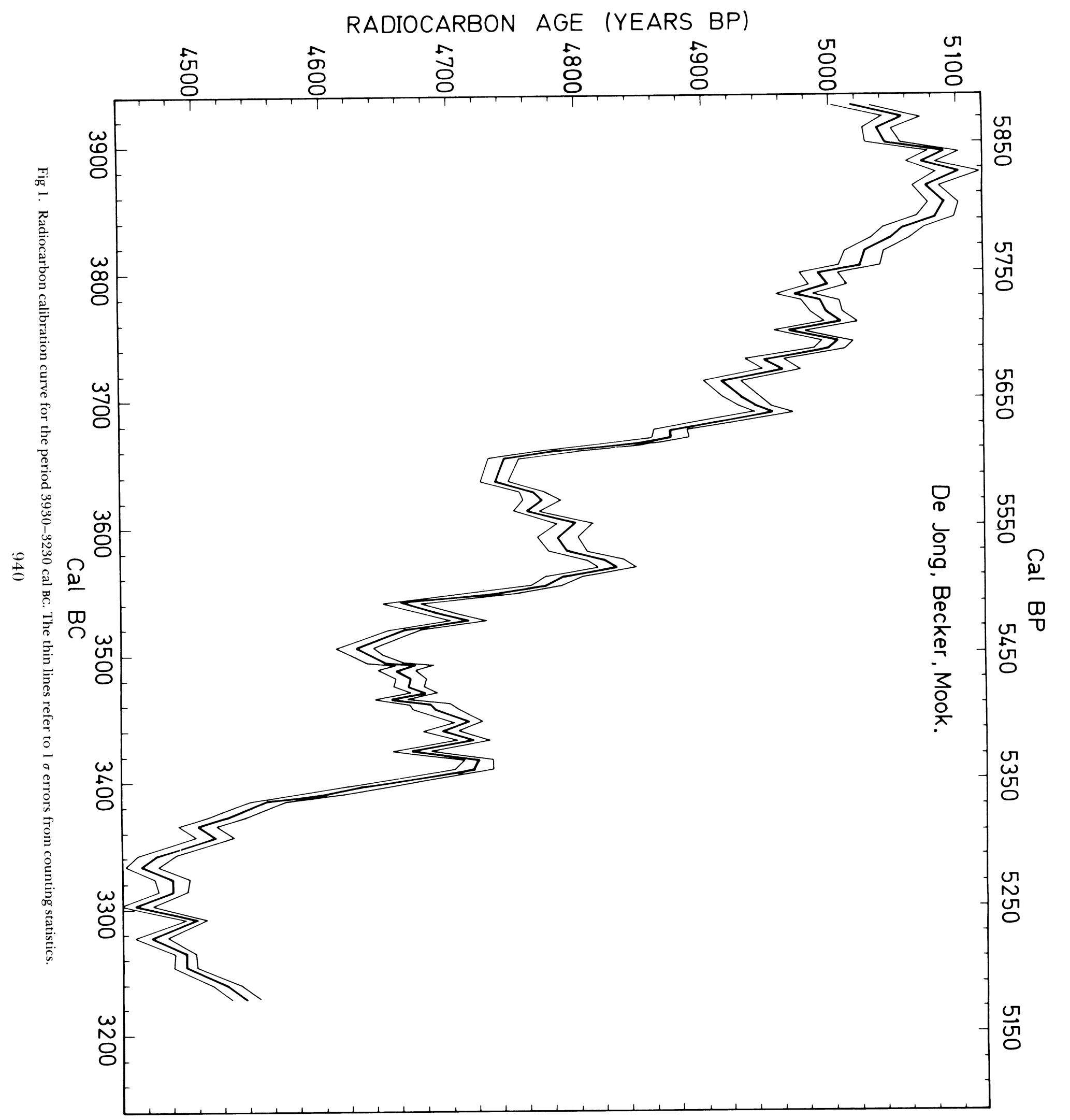


TABLE 1

Results of ${ }^{18} \mathrm{C}$ (vs $\mathrm{PDB}$ ) and ${ }^{14} \mathrm{C}$ analyses on tree rings from South German oak chronologies Donau 7, 3, 10, and 12. The absolute historical values are from the absolute master chronology (Becker \& Kromer, 1986). The ${ }^{14} \mathrm{C}$ results are conventional ages (5568 yr half-life), corrected for ${ }^{13} \mathrm{C}$.

\begin{tabular}{lccccc}
\hline $\begin{array}{l}\text { GrN } \\
\text { no. }\end{array}$ & $\begin{array}{c}\text { Tree } \\
\text { no. }\end{array}$ & $\begin{array}{c}\text { Tree-ring } \\
\text { no. }\end{array}$ & $\begin{array}{c}\text { Dendro } \\
\text { date BC }\end{array}$ & $\begin{array}{c}{ }^{14} \mathrm{C} \text { age } \\
(\mathrm{BP})\end{array}$ & $\begin{array}{c}\delta^{13} \mathrm{C} \\
(\%)\end{array}$ \\
\hline 9163 & E1 & 136 & 3930 & $5017 \pm 15$ & -26.18 \\
9162 & E1 & 145 & 3921 & $5057 \pm 15$ & -25.90 \\
9161 & E1 & 154 & 3912 & $5037 \pm 11$ & -26.01 \\
9160 & E1 & 165 & 3901 & $5043 \pm 15$ & -25.42 \\
9066 & E1 & 173 & 3893 & $5090 \pm 12$ & -25.51 \\
9065 & E1 & 181 & 3885 & $5072 \pm 11$ & -25.54 \\
9159 & F1 & 189 & 3877 & $5101 \pm 17$ & -24.50 \\
9063 & F1 & 200 & 3866 & $5076 \pm 11$ & -25.17 \\
9158 & G1 & 213 & 3853 & $5090 \pm 12$ & -26.16 \\
9025 & G1 & 224 & 3842 & $5083 \pm 15$ & -25.21 \\
9024 & H1 & 232 & 3834 & $5058 \pm 16$ & -25.05 \\
9023 & H1 & 241 & 3825 & $5047 \pm 15$ & -25.46 \\
9022 & H1 & 251 & 3815 & $5027 \pm 15$ & -25.36 \\
9021 & H1 & 262 & 3804 & $5023 \pm 16$ & -25.54 \\
9008 & H1 & 268 & 3798 & $4991 \pm 15$ & -25.55 \\
9007 & H1 & 277 & 3789 & $4998 \pm 15$ & -25.30 \\
9006 & H1 & 284 & 3782 & $4973 \pm 15$ & -25.37 \\
9005 & H1 & 289 & 3777 & $4992 \pm 15$ & -24.93 \\
9004 & H1 & 298 & 3768 & $4997 \pm 12$ & -25.06 \\
9002 & H1 & 306 & 3760 & $5008 \pm 13$ & -24.30 \\
9001 & H1 & 313 & 3753 & $4968 \pm 12$ & -24.65 \\
8837 & H1 & 321 & 3745 & $5006 \pm 12$ & -24.40 \\
8836 & H1 & 327 & 3739 & $4999 \pm 12$ & -24.54 \\
8835 & H1 & 335 & 3731 & $4948 \pm 15$ & -25.37 \\
8834 & H1 & 343 & 3723 & $4962 \pm 15$ & -24.53 \\
8833 & H1 & 352 & 3714 & $4915 \pm 15$ & -24.79 \\
8832 & H1 & 358 & 3708 & $4922 \pm 15$ & -24.73 \\
8831 & H1 & 365 & 3701 & $4930 \pm 15$ & -24.68 \\
8830 & H1 & 372 & 3694 & $4941 \pm 13$ & -24.53 \\
8779 & I1 & 377 & 3689 & $4955 \pm 15$ & -24.54 \\
8778 & I1 & 384 & 3682 & $4907 \pm 14$ & -24.82 \\
8777 & I1 & 390 & 3676 & $4874 \pm 13$ & -24.90 \\
8776 & I1 & 396 & 3670 & $4874 \pm 15$ & -24.59 \\
8775 & I1 & 400 & 3666 & $4853 \pm 12$ & -25.09 \\
8774 & I1 & 406 & 3660 & $4785 \pm 15$ & -24.84 \\
8773 & I1 & 412 & 3654 & $4743 \pm 12$ & -25.26 \\
8771 & I1 & 430 & 3636 & $4736 \pm 11$ & -25.27 \\
8766 & I1 & 438 & 3628 & $4765 \pm 20$ & -25.27 \\
8764 & I1 & 445 & 3621 & $4773 \pm 15$ & -24.81 \\
8751 & I1 & 453 & 3613 & $4761 \pm 10$ & -24.37 \\
8750 & K1 & 463 & 3603 & $4799 \pm 14$ & -24.49 \\
8749 & K1 & 474 & 3592 & $4785 \pm 16$ & -24.69 \\
8748 & K1 & 485 & 3581 & $4793 \pm 15$ & -24.68
\end{tabular}

TABLE 1 (continued)

\begin{tabular}{|c|c|c|c|c|c|}
\hline $\begin{array}{l}\text { GrN } \\
\text { no. }\end{array}$ & $\begin{array}{l}\text { Tree } \\
\text { no. }\end{array}$ & $\begin{array}{c}\text { Tree-ring } \\
\text { no. }\end{array}$ & $\begin{array}{l}\text { Dendro } \\
\text { date BC }\end{array}$ & $\begin{array}{c}{ }^{14} \mathrm{C} \text { age } \\
\text { (BP) }\end{array}$ & $\begin{array}{l}\delta^{13} \mathrm{C} \\
\left(\%{ }^{\circ}\right)\end{array}$ \\
\hline 8742 & $\mathrm{~K} 1$ & 492 & 3574 & $4822 \pm 15$ & -24.76 \\
\hline 8741 & $\mathrm{Kl}$ & 498 & 3568 & $4831 \pm 15$ & -24.65 \\
\hline 8740 & $\mathrm{~K} 1$ & 505 & 3561 & $4790 \pm 14$ & -25.06 \\
\hline 8730 & $\mathrm{~K} 1$ & 512 & 3554 & $4775 \pm 12$ & -24.90 \\
\hline 8729 & $\mathrm{~K} 1$ & 518 & 3548 & $4739 \pm 15$ & -24.60 \\
\hline 8727 & $\mathrm{~K} 1$ & 525 & 3541 & $4662 \pm 15$ & -24.43 \\
\hline 8726 & $\mathrm{~K} 1$ & 532 & 3534 & $4688 \pm 14$ & -25.31 \\
\hline 8725 & $\mathrm{~K} 1$ & 539 & 3527 & $4714 \pm 14$ & -25.21 \\
\hline 8724 & L1 & 546 & 3520 & $4664 \pm 11$ & -25.02 \\
\hline 8570 & M1 & 553 & 3513 & $4644 \pm 13$ & -25.54 \\
\hline 8569 & M1 & 560 & 3506 & $4625 \pm 14$ & -24.71 \\
\hline 8568 & M1 & 566 & 3500 & $4635 \pm 12$ & -25.03 \\
\hline 8549 & Ml & 572 & 3494 & $4649 \pm 15$ & -26.12 \\
\hline 8728 & $\mathrm{Nl}$ & 574 & 3492 & $4672 \pm 15$ & -23.99 \\
\hline 8548 & $\mathrm{Ol}$ & 578 & 3488 & $4658 \pm 15$ & -25.66 \\
\hline 8547 & $\mathrm{Ol}$ & 585 & 3481 & $4669 \pm 12$ & -25.69 \\
\hline 8546 & $\mathrm{Ol}$ & 591 & 3475 & $4667 \pm 12$ & -25.58 \\
\hline 8532 & $\mathrm{O} 1$ & 596 & 3470 & $4679 \pm 11$ & -25.09 \\
\hline 8531 & $\mathrm{O} 1$ & 601 & 3465 & $4654 \pm 13$ & -24.60 \\
\hline 8530 & $\mathrm{P} 1$ & 605 & 3461 & $4684 \pm 16$ & -25.33 \\
\hline 8529 & P1 & 609 & 3457 & $4688 \pm 17$ & -24.95 \\
\hline 8528 & Q1 & 614 & 3452 & $4702 \pm 15$ & -24.98 \\
\hline 8527 & Q1 & 619 & 3447 & $4714 \pm 11$ & -25.13 \\
\hline 8524 & Q1 & 626 & 3440 & $4693 \pm 15$ & -25.77 \\
\hline 8523 & Q1 & 633 & 3433 & $4718 \pm 13$ & -25.35 \\
\hline 8522 & $\mathrm{R} 1$ & 641 & 3425 & $4669 \pm 15$ & -25.46 \\
\hline 8521 & $\mathrm{Rl}$ & 649 & 3417 & $4722 \pm 11$ & -25.26 \\
\hline 8520 & $\mathrm{R} 1$ & 656 & 3410 & $4718 \pm 15$ & -25.63 \\
\hline 8475 & $\mathrm{R} 1$ & 661 & 3405 & $4692 \pm 13$ & -25.44 \\
\hline 8474 & S1 & 669 & 3397 & $4631 \pm 13$ & -25.46 \\
\hline 8473 & Sl & 676 & 3390 & $4600 \pm 12$ & -25.27 \\
\hline 8472 & S1 & 687 & 3385 & $4556 \pm 14$ & -24.84 \\
\hline 8366 & S1 & 694 & 3372 & $4522 \pm 15$ & -24.79 \\
\hline 8365 & $\mathrm{~T} 1$ & 700 & 3366 & $4500 \pm 15$ & -24.20 \\
\hline 8346 & $\mathrm{~T} 1$ & 709 & 3357 & $4514 \pm 15$ & -24.43 \\
\hline 8345 & $\mathrm{~T} 1$ & 723 & 3343 & $4468 \pm 15$ & -24.05 \\
\hline 8299 & $\mathrm{~T} 1$ & 732 & 3334 & $4456 \pm 13$ & -24.81 \\
\hline 8298 & U1 & 742 & 3324 & $4480 \pm 14$ & -24.98 \\
\hline 8273 & $\mathrm{~V} 1$ & 752 & 3314 & $4480 \pm 11$ & -24.53 \\
\hline 8272 & $\mathrm{Vl}$ & 763 & 3303 & $4451 \pm 13$ & -25.37 \\
\hline 8271 & Vl & 774 & 3292 & $4499 \pm 8$ & -24.57 \\
\hline 8270 & W1 & 788 & 3278 & $4463 \pm 13$ & -24.67 \\
\hline 8269 & Wl & 801 & 3265 & $4490 \pm 8$ & -24.41 \\
\hline 8268 & W1 & 812 & 3254 & $4490 \pm 9$ & -25.28 \\
\hline 8267 & W1 & 826 & 3240 & $4523 \pm 11$ & -25.03 \\
\hline 8266 & W1 & 837 & 3229 & $4538 \pm 11$ & -25.28 \\
\hline
\end{tabular}

